\title{
Variación fenotípica de la langosta espinosa Panulirus argus (Decapoda: Paniluridae) en el Caribe Suroccidental
}

\author{
Nacor Bolaños-Cubillos ${ }^{1,2}$, Néstor H. Campos ${ }^{2}$ \& Edna J. Márquez ${ }^{1}$
}

1. Corporación para el Desarrollo Sostenible del Archipiélago de San Andrés, Providencia y Santa Catalina, CORALINA Colombia; nacorwbc@yahoo.com,nwbolanosc@unal.edu.co

2. Universidad Nacional de Colombia Sede Caribe, CECIMAR, Calle 25 No. 2-55, Playa Salguero, Santa Marta D.T.C.H., Colombia; nhcamposc@unal.edu.co

3. Universidad Nacional de Colombia-Sede Medellín-Facultad de Ciencias, Calle 59A No $63-2018$ Bloque 19 A Laboratorio 310, Medellín, 050034, Colombia; ejmarque@unal.edu.co, ejmarque@gmail.com

$$
\text { Recibido 07-IX-2015. Corregido 21-III-2016. Aceptado 15-IV-2016. }
$$

\begin{abstract}
Phenotypic variation of Panulirus argus (Decapoda: Paniluridae) in Southwest Caribbean. The spiny lobster Panulirus argus is an important fishery species in the Western Central Atlantic. Changes in the dispersion range through its life cycle and heterogeneous habitats, suggest that $P$. argus exhibit phenotypic plasticity. However, the morphometric variation of this species is unknown so far, although this information is relevant in evolutionary studies as well as to solve questions of fishery interest. Thus, the aim of this study was to determine whether $P$. argus exhibit phenotypic variation between sexes, among five geographic origins and three oceanographic conditions of Southwest Caribbean (Colombian archipelago San Andrés, Providencia y Santa Catalina). A total of 193 P. argus adults were submitted to geometric morphometrics using six landmarks that delimit one half of the sternal plate. The differences in sternal plate size were compared with KruskalWallis and Mann-Whitney Tests. The allometric effect was estimated using Multivariate Regression Analysis, the model of allometric slopes was tested by Multivariate analysis of covariance and the sternal plate shape differences was explored using non-parametric comparisons of Euclidian distances and Neighbour Joinnig trees. The results showed that the morphometric variation of sternal plate of this spiny lobster varied according to the gender since the sexual size and shape dimorphisms were significant. In both sexes, the sternal plate shape differed among oceanographic scenarios as it was evidenced by significant differences among Euclidian distances, and the tendency to cluster by North, Centre and South sections of San Andrés archipelago. Additionally, the morphometric variation resulting from phenotypic plasticity to variable ecological contexts may explain the phenotypic differences among genetically similar populations. This information permits to define management units, support the selection of regulatory policies of this fishery and complement the genetic analysis of the species in this Caribbean region. Rev. Biol. Trop. 64 (3): 975-990. Epub 2016 September 01.
\end{abstract}

Key words: geometric morphometrics, geometric morphology, phenotypic plasticity, Caribbean spiny lobster, phenotypic stocks, fishery management.

La langosta espinosa Panulirus argus (Latreille, 1804) es un crustáceo decápodo perteneciente a la familia Palinuridae (Tavares, 2002) que se encuentra distribuida desde Carolina del Norte hasta Río de Janeiro, incluyendo el Golfo de México y el Mar Caribe (Holthuis, 1991; Tavares, 2002). Esta especie constituye uno de los recursos pesqueros de mayor valor económico y ecológico de todo el Caribe (Goldstein, 2004). Su complejo ciclo de vida incluye cinco fases, una de las cuales es planctónica con una duración aproximada entre cinco y doce meses, dependiendo de las condiciones ambientales (Lewis, 1951; Silberman, Sarver, \& Walsh, 1994); posteriormente, se asienta y continua su vida juvenil y adulta en los lugares de arribo, que normalmente se encuentran en aguas someras, pero pueden 
alcanzar alrededor de los $90 \mathrm{~m}$ de profundidad (FAO, 2001).

La información sobre variación fenotípica de langostas es escasa y completamente desconocida para $P$. argus. A la fecha solo se han descrito estudios morfométricos en Homarus americanus (Cadrin, 1995; Harding, Kenchington, \& Zheng, 1993), Homarus gammarus (Debuse, Addison, \& Reynodls, 2001), Palinurus elephas (Tidu et al., 2004) y Panulirus inflatus (García-Rodríguez, Aguero, Perez-Enriquez, \& MacLeod, 2004). En su conjunto, estos estudios han mostrado que las langostas pueden exhibir dimorfismo sexual, alometría, diferencias inter-poblacionales y plasticidad fenotípica. Hallazgos similares se han encontrado en estudios morfométricos de otros crustáceos tales como Aegla marginata (Trevisan et al., 2012), Austropotamobius pallipes (Bertocchi, Brusconi, Gherardi, Buccianti, \& Scalici, 2008), Carcinus maenas (Ledesma, Van der Molen, \& Barón , 2010; Silva, Alves, Paula, \& Hawkins, 2010; Souza , Ilarri, Campos, Marques, \& Martins, 2011), Cyrtograpsus affinis (Lezcano, González, Spivak, \& Dellatorre, 2012), Cyrtograpsus angulatus (Idaszkin, Márquez, \& Nocera, 2013), Liocarcinus depurator (Rufino, Abelló, \& Yule, 2004; Guerao \& Abelló, 2011), Munida gregaria (Pérez-Barros, Calcagno, \& Lovrich, 2011), Munida rugosa (Claverie \& Smith, 2010) y Ucides cordatus (Alencar, Lima-Filho, Molina, $\&$ Freire. 2014), entre otros.

Además de su papel en contextos evolutivos, los análisis morfométricos proveen información de clanes (stocks) fenotípicos, los cuales designan grupos de individuos con similares tasas de crecimiento, mortalidad y reproducción (Booke, 1981). Tal información permite determinar cómo cada stock responde a la explotación debido a que las tasas ontogénicas, a pesar de estar afectadas por el ambiente, afectan muchos atributos de la población que pueden estar íntimamente relacionadas a la dinámica poblacional (Garrod \& Horwood, 1984). De esta manera, los grupos con diferentes dinámicas de crecimiento y reproducción deben ser modulados y manejados separadamente, independiente de su homogeneidad genética (Cadrin, 2005).
Lo anterior es relevante para $P$. argus, una especie con prolongada duración larvaria, lo cual sugiere un extensivo intercambio génico entre sitios. Esta idea ha sido corroborada por estudios genéticos de $P$. argus en el Océano Atlántico, los cuales, utilizando diferentes marcadores moleculares como isoenzimas (Hateley \& Sleeter, 1992), RFLPs del DNA mitocondrial (Silberman et al., 1994), rRNA 16S, COI, región control (J. D. Rangel-Medrano, comunicación personal, 15 de diciembre de 2015) y microsatélites (Truelove, Box, Butler, Behringer, \& Preziosi, 2013), han mostrado generalmente flujo génico. En este contexto, la variación fenotípica de la langosta puede aportar información complementaria para la identificación de unidades de monitoreo y manejo de este recurso en varios sectores del Caribe.

En el Caribe suroccidental, el archipiélago colombiano de San Andrés, Providencia y Santa Catalina (ASPSC) limita con varios países como Panamá, Costa Rica, Nicaragua, Honduras y Jamaica, en un sector donde confluyen diversos sistemas de corrientes que pueden jugar un papel importante en la dispersión de larvas de varias especies. En este archipiélago, $P$. argus representa el principal producto pesquero de exportación (Castro, 2008) y aporta aproximadamente el $80 \%$ de la producción de Colombia y el $2 \%$ de la producción total en el Caribe (Cruz, 2002). Sin embargo, este recurso mantiene una pesquería industrial importante desde la década de 1980, razón por la cual se ha sugerido que puede estar sometido a sobreexplotación (FAO, 2007).

Para evitar detrimentos por sobrepesca, las decisiones e implementación de algunas medidas de manejo para la pesquería de langosta se han apoyado en varios estudios biológicos y pesqueros del recurso en esta área (Arango, Box, Butler, Behringer, \& Preziosi, 2001; Prada, Castro, Mitchell, \& Bent, 2007). Además, se han adelantado estudios que indican similitud genética entre poblaciones de diferentes sitios del archipiélago (J. D. RangelMedrano, comunicación personal, diciembre 15 de 2015). Sin embargo, se desconoce si la langosta espinosa podría estar representada por 
diferentes stocks fenotípicos dada la heterogeneidad ambiental de la zona.

Varias observaciones sugieren que las langostas espinosas de esta región del Caribe, pueden presentar variaciones fenotípicas entre cayos, bancos y bajos del ASPSC. Debido a la batimetría de la zona, no existe dispersión de individuos jóvenes y adultos de $P$. argus entre sitios; lo que indica que gran parte del desarrollo desde la postlarva hasta adulto está confinado al lugar donde se haya asentado la larva. Tales sitios pueden representar hábitats con diferentes grados de presión natural por diferencias promovidas por los sistemas de corrientes del sector (Garay et al., 1988; Molares, Vanegas, Bustamante, \& Andrade, 2004). Además, algunos sitios están sometidos a pesca artesanal mientras que otros están sometidos a pesca industrial, generando una presión antrópica diferencial entre estas áreas. Finalmente, en este sector se han observado diferencias morfométricas que pueden ser explicadas tanto por diferencias genéticas como por plasticidad fenotípica en otra especie con larvas pelágicas de duración relativamente larga (1 mes) como Strombus gigas (Márquez et al., 2013; E. J. Márquez, comunicación personal, diciembre 15 de 2015).

El objetivo de este estudio fue determinar si existe variación fenotípica de la placa esternal de la langosta espinosa Panulirus argus en el ASPSC, Reserva de Biosfera Seaflower, Caribe sur occidental. Se utilizó Morfometría Geométrica basada en puntos anatómicos de referencia (landmarks) para evaluar si el tamaño y la conformación de la placa esternal de la langosta espinosa difieren de acuerdo al sexo, al origen geográfico y al escenario oceanográfico. Esta información complementa los estudios biológicos, pesqueros y genéticos que contribuyen a la identificación de unidades de monitoreo y manejo de este recurso.

\section{MATERIALES Y MÉTODOS}

Muestreo y área de estudio: Se recolectaron manualmente 281 langostas, mediante buceo a pulmón libre y equipos autónomos a menos de $30 \mathrm{~m}$ de profundidad. Las muestras provenían de nueve sitios separados por grandes distancias (entre $30 \mathrm{~km}$ y $496 \mathrm{~km}$ aproximadamente) y profundidades variables hasta de $3000 \mathrm{~m}$, en la zona norte (Serranilla, Bajo Alicia, Bajo Nuevo, Serrana, Roncador), centro (Providencia) y sur (San Andrés, Bolívar, Albuquerque) del archipiélago de San Andrés, Providencia y Santa Catalina (ASAPSC), en el Caribe suroccidental (Fig. 1).

Esta zona presenta heterogeneidad ambiental debido a la influencia de la Corriente Central del Caribe (zona norte), giro Panamá-Colombia (zonas centro y sur), corrientes superficiales con distribución no homogénea (Richardson, 2005), remolinos ciclónicos que facilitan el ascenso de aguas profundas más frías y ricas en nutrientes en algunos sectores (Andrade-Amaya, 2012) y la presencia de un surco profundo entre las islas de San Andrés y Providencia (Andrade, 2001; Molares et al., 2004). Adicionalmente, presenta diferencias en las coberturas coralinas, complejidades de los ecosistemas arrecifales y especies asociadas (Sánchez et al., 2005; Abril-Howard, Orozco-Toro, BolañosCubillos, \& Bent-Hooker, 2012), y diferentes presiones de pesca artesanal (Bolívar y Providencia) e industrial o mixta (Bajo Alicia, Bajo Nuevo, Serranilla y Serrana).

Morfometría geométrica: Para el registro fotográfico, cada espécimen completo se colocó en la misma posición, ángulo y dirección sobre una superficie plana y con un metro como escala de referencia. Las fotografías se tomaron con una cámara SONY DSC -W70 a una distancia focal de $90 \mathrm{~cm}$ procurando que la placa esternal quedara en el centro del campo visual para evitar distorsiones ópticas periféricas.

En cada fotografía se identificaron cuatro puntos anatómicos de referencia (en inglés, landmarks) Tipo I y dos puntos Tipo III (Bookstein, 1991) sobre la mitad derecha de la placa esternal. Los puntos Tipo I se ubicaron en la inserción de los primeros cuatro pares de apéndices caminadores. Los dos puntos Tipo III correspondieron, respectivamente, a la mitad de las distancias entre los sitios de inserción 


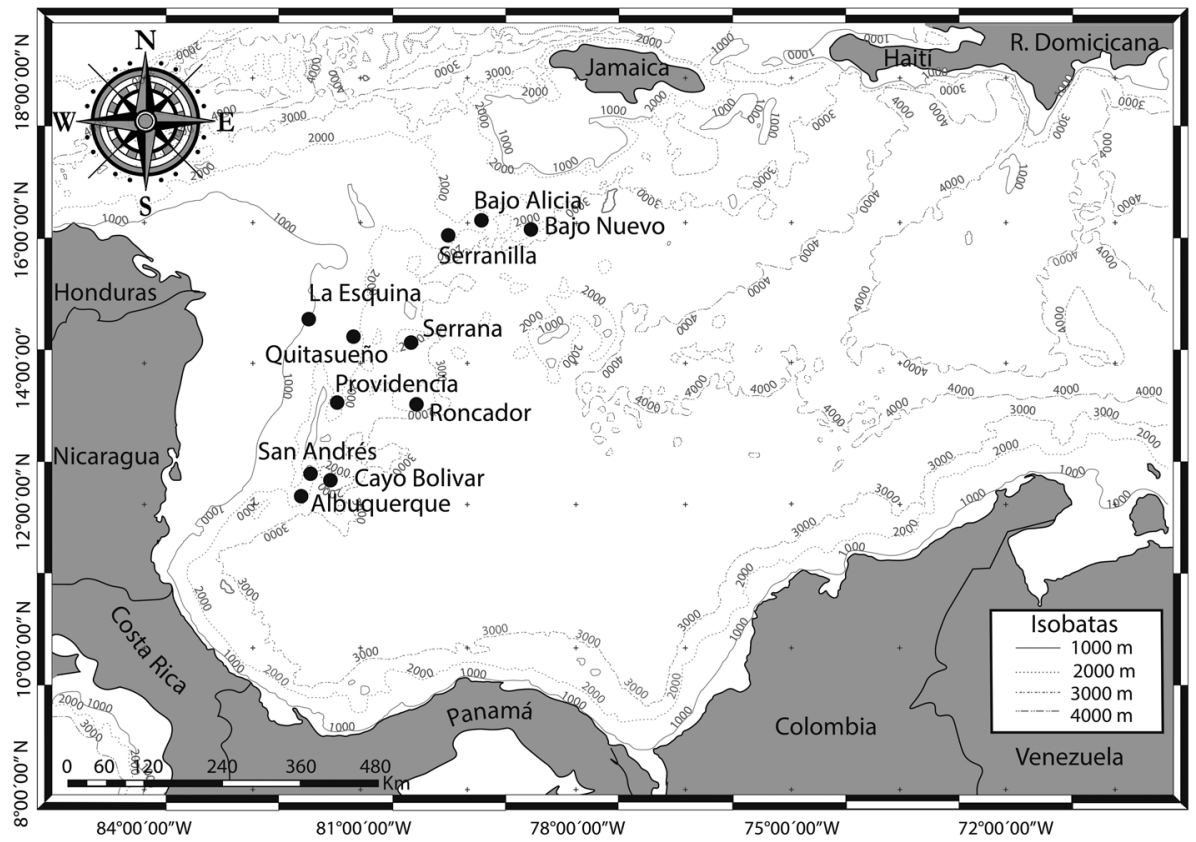

Fig. 1. Sitios de muestreo de P. argus en el archipiélago de San Andrés, Providencia y Santa Catalina.

Fig. 1. Sampling sites of $P$. argus in the archipelago San Andrés, Providencia y Santa Catalina.

izquierda y derecha de las patas caminadoras 1 y 4 (Fig. 2). Estos puntos anatómicos cumplen con los criterios descritos por Klingenberg, Barluenga, \& Meyer (2002) y Zelditch, Donald, Swiderski, Sheets, \& Fink (2004), respecto a homología, cobertura y distribución regular sobre la estructura completa, posición sobre el mismo plano y no redundancia.

La digitalización de las coordenadas (COO), la repetibilidad en la toma de puntos anatómicos de referencia (VAR) y la generación de las variables de tamaño y conformación (MOG) se realizaron con los diferentes módulos del paquete CLIC V70 (Dujardin, 2013). Para estimar la precisión de los puntos de referencia en los individuos, las coordenadas se digitalizaron dos veces sobre 281 fotos y se evaluó la repetibilidad, la cual se calcula como la varianza individual/varianza total en una ANOVA de una vía, modelo II sobre medidas repetidas (Arnqvist \& Märtensson, 1998).
Para obtener las variables de tamaño y conformación, las coordenadas crudas se sometieron al Análisis Generalizado de Procrustes. En este análisis, la suma de las distancias elevadas al cuadrado entre cada uno de los objetos y la configuración del objeto de referencia se minimiza repetidamente por traslaciones y rotaciones rígidas (Rohlf, 1990; Goodall, 1991). Este procedimiento elimina los efectos de la escala, posición y orientación en el espacio de los objetos bajo estudio, generando variables de conformación libres de la variación no biológica.

Como variable de tamaño, se utilizó el estimador isométrico conocido como Tamaño Centroide, el cual se define como la raíz cuadrada de la suma de los cuadrados de las distancias de todos los puntos de referencia a su centroide (Klingerberg et al., 2002). Como variables de conformación, se utilizaron: (1) los componentes no uniformes (deformaciones parciales o en inglés, partial warps), los cuales 


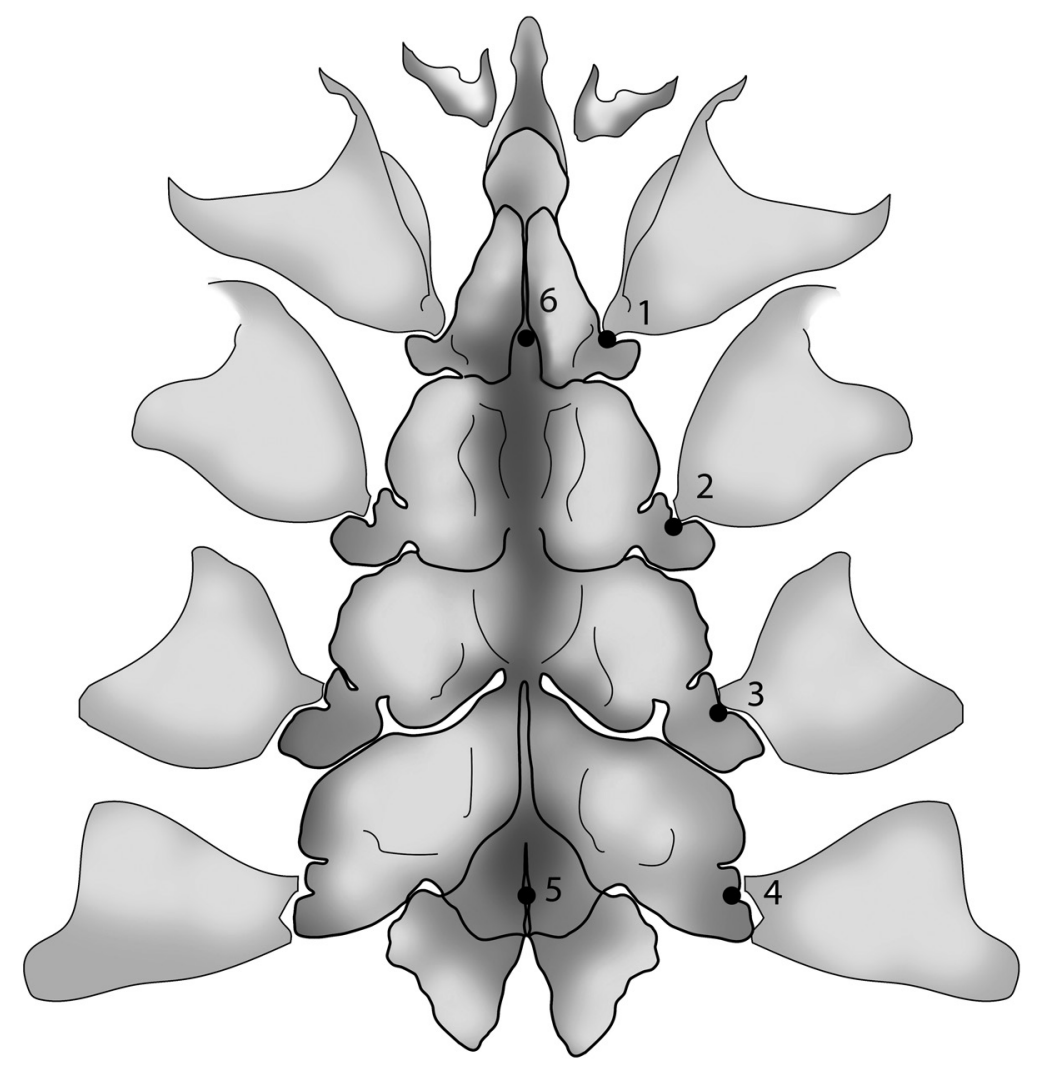

Fig. 2. Puntos anatómicos de referencia de la placa esternal de P. argus. La numeración de los puntos de referencia denota el arreglo seguido durante la digitalización.

Fig. 2. Landmarks on the P. argus sternal plate. Numbering of landmarks denote the arrangement followed in the coordinate digitalization.

representan las deformaciones regionales de cada objeto respecto al de la referencia y (2) los componentes uniformes, los cuales representan las deformaciones globales (Bookstein, 1991).

Para los análisis estadísticos se utilizaron 193 langostas adultas (64 hembras y 129 machos) debido a que se excluyeron datos de individuos jóvenes para evitar diferencias asociadas al estado de desarrollo, hembras con espermateca que impidieron la observación de puntos anatómicos de referencia y sitios que exhibieron bajo número muestral (Bajo Alicia, Roncador, San Andrés y Albuquerque). La comparación del tamaño entre sexos, entre sitios y entre escenarios oceanográficos, se realizó con la prueba de Kruskall-Wallis y las comparaciones pareadas de Mann Whitney debido a que la variable no satisfizo los supuestos requeridos para aplicar una prueba paramétrica.

La comparación de la conformación de la placa esternal entre sexos y entre orígenes geográficos se realizó mediante comparación de las distancias Euclidianas entre los grupos comparados, debido a que no se cumplieron los supuestos de normalidad y homocedasticidad multivariadas. La significancia estadística de las diferencias entre las distancias Euclidianas en cada uno de los contrastes, se calculó con 1000 permutaciones y se ajustó por Bonferroni en las comparaciones múltipes (Rice, 1989).

El efecto residual del tamaño en las variables de conformación, se exploró utilizando un análisis de regresión multivariado y una prueba de permutación para la significancia 
estadística (Good, 2000). Para averiguar si las pendientes alométricas seguían un patrón común, se utilizó un análisis multivariado de covarianza. En todos los contrastes en los que se encontró efecto alométrico significativo con pendientes similares, se calculó la significancia estadística después de la corrección por tamaño. Finalmente, las distancias Euclidianas entre sitios se utilizaron para construir un dendrograma con el método de unión de vecinos (Neighbour Joining).

\section{RESULTADOS}

La repetibilidad de las mediciones fue confiable para las coordenadas alineadas (0.8610.990), el tamaño $(\mathrm{R}=0.999)$ y la conformación para los primeros cinco componentes principales (0.866-0.992) los cuales representan el 94 $\%$ de la variación total.

Variación del tamaño: Se detectaron diferencias significativas del tamaño de la placa esternal entre hembras y machos de los sitios evaluados (Kruskal-Wallis $=63.91, \mathrm{P}<0.001)$.
En la comparación dentro de cada sitio, las diferencias del tamaño de la placa esternal entre sexos fueron significativas en Providencia y Serrana pero no lo fueron en el atolón Bolívar, Serranilla y Bajo Nuevo (Cuadro 1).

La comparación del tamaño por origen geográfico mostró diferencias significativas tanto en machos (Kruskal-Wallis= 29.69, $\mathrm{P}<0.001)$ como en hembras (Kruskal-Wallis= 24.02, $\mathrm{P}<0.001)$ pero no se observó una tendencia clara. Por ejemplo, los machos de Serranilla y Providencia fueron mucho más pequeños que los de Bajo Nuevo, Serrana y Bolívar (Fig. 3). De otro lado, las hembras solo conformaron dos grupos de tamaños en los que las langostas de Bolívar y Bajo Nuevo fueron las más grandes (Fig. 3).

Entre zonas, el tamaño promedio de los machos de la zona centro fue significativamente más pequeño que los de las zonas norte $(\mathrm{P}=$ $0.005)$ y $\operatorname{sur}(\mathrm{P}=0.010)$ (Fig. 4). Las hembras de la zona sur fueron significativamente más grandes que las de la zona centro $(\mathrm{P}<0.001)$ pero no mostraron diferencias con la zona norte $(\mathrm{P}=0.027)$ después de la corrección

\section{CUADRO 1}

Estadística descriptiva del tamaño de la placa esternal en hembras y machos de $P$. argus en el archipiélago de San Andrés, Providencia y Santa Catalina

TABLE 1

Descriptive statistics of the sternal plate size in females and males of $P$. argus in the San Andrés, Providencia and Santa Catalina archipelago

\begin{tabular}{|c|c|c|c|c|c|c|}
\hline Sitio & Sexo & $\mathrm{N}$ & Media & $\mathrm{DE}$ & Mediana & $\mathrm{P}$ \\
\hline \multirow[t]{2}{*}{ Bolívar } & Hembra & 14 & 5.963 & 0.381 & 6.004 & 0.968 \\
\hline & Macho & 28 & 6.180 & 1.152 & 6.014 & \\
\hline \multirow[t]{2}{*}{ Providencia } & Hembra & 33 & 4.992 & 0.512 & 4.943 & $0.002 *$ \\
\hline & Macho & 41 & 5.433 & 0.585 & 5.402 & \\
\hline \multirow[t]{2}{*}{ Serrana } & Hembra & 7 & 4.759 & 1.059 & 4.310 & $0.008 *$ \\
\hline & Macho & 29 & 6.197 & 1.022 & 6.361 & \\
\hline \multirow[t]{2}{*}{ Serranilla } & Hembra & 5 & 4.857 & 1.134 & 4.488 & 0.097 \\
\hline & Macho & 15 & 5.113 & 0.639 & 4.789 & \\
\hline \multirow[t]{2}{*}{ Nuevo } & Hembra & 5 & 5.899 & 0.787 & 5.877 & 0.231 \\
\hline & Macho & 16 & 6.374 & 0.871 & 6.651 & \\
\hline
\end{tabular}

$\mathrm{N}$ : tamaño muestral, DE: desviación estándar, P: significancia estadística de las diferencias de tamaño entre machos y hembras. El asterisco denota diferencias estadísticamente significativas.

$\mathrm{N}$ : sample size, DE: standard deviation, P: statistical significance of size between males and females. Asterisks denote significant statistical differences. 

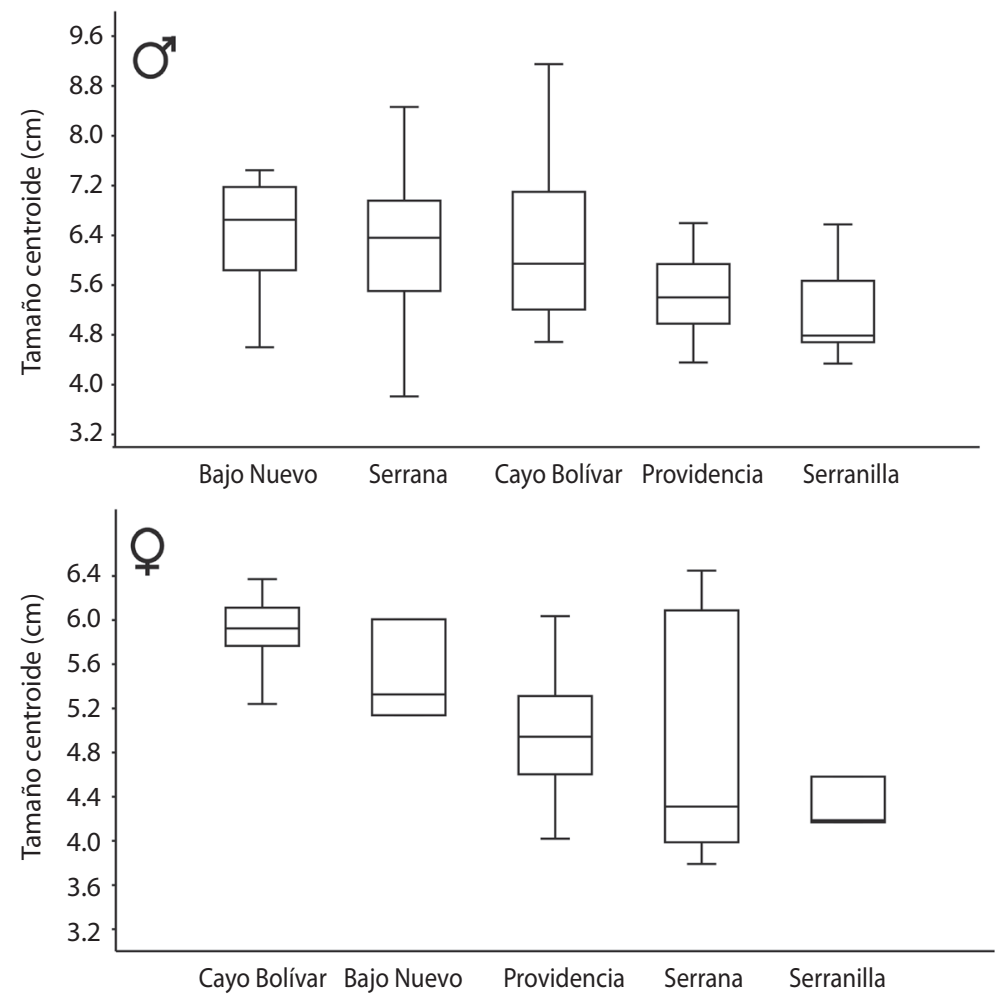

Fig. 3. Variación del tamaño (cm) de machos y hembras adultos de P. argus entre sitios.

Fig. 3. Size variation $(\mathrm{cm})$ of male and female adults of $P$. argus among geographical sites.
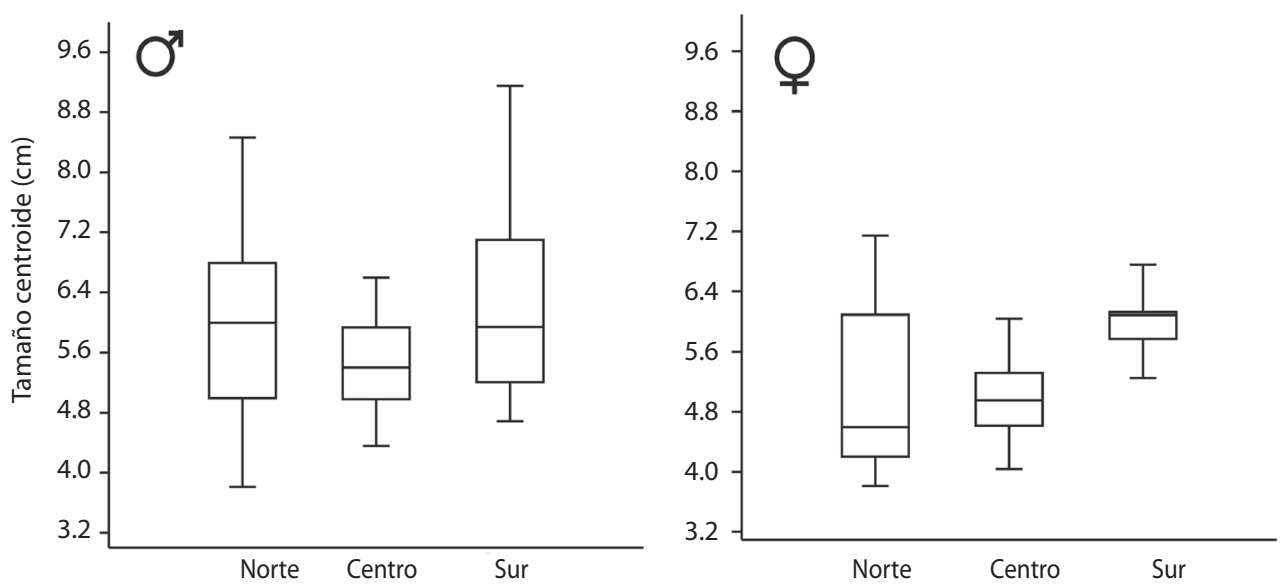

Fig. 4. Variación del tamaño $(\mathrm{cm})$ de machos y hembras de $P$. argus de acuerdo con el escenario oceanográfico del archipiélago de San Andrés.

Fig. 4. Size variation $(\mathrm{cm})$ of males and females of $P$. argus among oceanographic scenarios of San Andrés archipelago. 
Bonferroni. Entre las hembras de las zonas centro y norte no se detectaron diferencias estadísticas significativas ( $\mathrm{P}=0.774)$ (Fig. 4).

Variación de la conformación: Las diferencias de conformación de la placa esternal entre sexos fueron significativas en los sitios examinados (Cuadro 2). El análisis del dimorfismo sexual incluyendo o removiendo el efecto alométrico mostró diferencias significativas en todos sitios (Cuadro 2).

La comparación de las configuraciones promedio de la placa esternal entre machos y hembras mostró que la mayor variación estuvo concentrada entre los puntos de referencia 4 y 5 , los cuales representan la mitad de la placa esternal delimitada por los puntos de inserción del cuarto par de patas caminadoras (pleópodos). En esta sección las hembras presentan modificados los esternitos sexto y séptimo en forma de receptáculo seminal denominado espermateca. Estas diferencias se mantuvieron para todos los sitios (Fig. 5a).

La comparación de la conformación por sitio, mostró una tendencia de agrupación de acuerdo a la zona del ASPSC: norte, centro y sur (Fig. 6). Esta tendencia de agrupación es concordante con los escenarios oceanográficos definidos por la influencia de la corriente central del Caribe y el giro Panamá-Colombia (Fig. 6). Para ambos sexos, las diferencias de las distancias Euclidianas de las conformaciones de las placas esternales fueron estadísticamente significativas entre las zonas (Cuadro 3 ).

La comparación de las configuraciones promedio de la placa esternal entre sitios mostró que la conformación de la parte anterior de la placa esternal (puntos de referencia 2, 3,5 y 6) de los machos fue más estrecha en el sector norte, seguido del sector centro y sur (Fig. 5b). Un resultado similar se encontró en las hembras excepto en Serrana, donde las conformaciones promedio fueron ligeramente menos estrechas que las de Serranilla y Nuevo (Fig. 5b). Lo anterior muestra un gradiente en el que la placa esternal se va estrechando en el sentido sur - norte. Los cambios por escenario oceanográfico están relacionados con los puntos de inserción de los pares de patas caminadoras 2 y 3 .

\section{DISCUSIÓN}

En este trabajo se determinó la variación fenotípica de la placa esternal de la langosta espinosa $P$. argus en el ASPSC debido al sexo, la localización geográfica y el escenario oceanográfico. Los resultados mostraron que todos

\section{CUADRO 2}

Distancias Euclidianas entre la conformación de machos y hembras de P. argus en el archipiélago de San Andrés, calculadas con y sin efecto alométrico

TABLE 2

Euclidian distances between males and females of $P$. argus from the San Andrés archipelago, calculated with and without allometric effect

\begin{tabular}{llcccccc}
\multicolumn{1}{r}{ Sitio } & \multicolumn{2}{c}{$\mathrm{N}$} & \multicolumn{2}{c}{ Con alometría } & \multicolumn{2}{c}{ Sin alometría } \\
Nuevo & $\mathrm{M}$ & $\mathrm{H}$ & $\mathrm{DEu}$ & $\mathrm{P}$ & $\mathrm{DEu}$ & $\mathrm{P}$ \\
Serranilla & 16 & 5 & 0.028 & $0.002^{*}$ & 0.022 & $0.001^{*}$ \\
Serrana & 15 & 5 & 0.041 & $\mathrm{~ns}$ & 0.041 & $0.000^{*}$ \\
Providencia & 29 & 7 & 0.045 & $0.000^{*}$ & 0.024 & $0.019 *$ \\
Bolívar & 41 & 33 & 0.029 & $0.000^{*}$ & 0.023 & $0.000^{*}$ \\
\hline
\end{tabular}

DEu: Distancias Euclidianas, N: número muestral, M: machos, H: hembras, ns: no significativo. El asterisco denota diferencias estadísticamente significativas.

DEu: Euclidian distances, N: Sample size, M: males, H: females, ns: non-significant. Asterisks denote significant statistical differences. 
a.

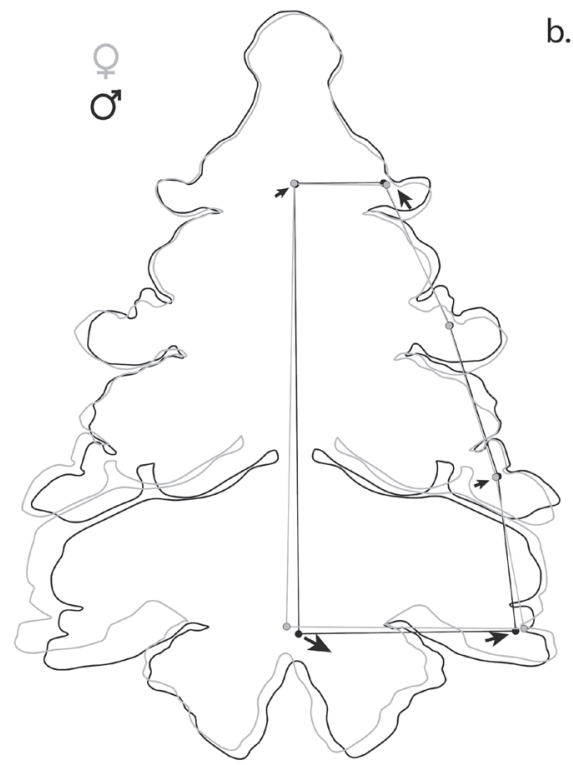

b. Cayo Bolívar

- Providencia

- Serrana

- Serranilla-Bajo

Nuevo

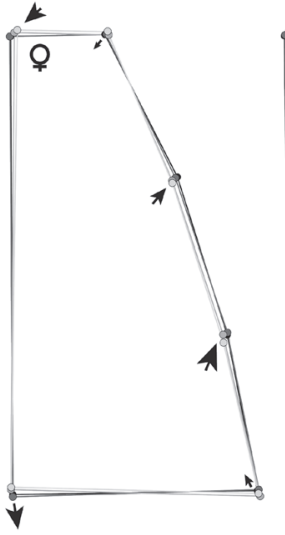

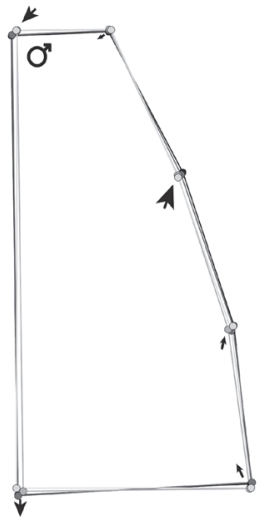

Fig. 5. a. Dimorfismo sexual en la conformación de la placa esternal de $P$. argus. b. Diferencias en la conformación de machos y hembras entre escenarios oceanográficos. Los polígonos conectan las coordenadas residuales después de la superposición de Procrustes al consenso general. Los círculos sólidos representan los puntos anatómicos de referencia (landmarks). Las flechas indican los puntos de mayor variación en la conformación de la placa esternal.

Fig. 5. a. Sexual shape dimorphism of sternal plate of $P$. argus. b. Shape differences of males and females among oceanographic scenarios. Polygons connect the residual coordinates after Procrustes superimposition to the general consensus. Solid circles represent landmarks. Arrows indicate the highest differences in sternal plate shape.

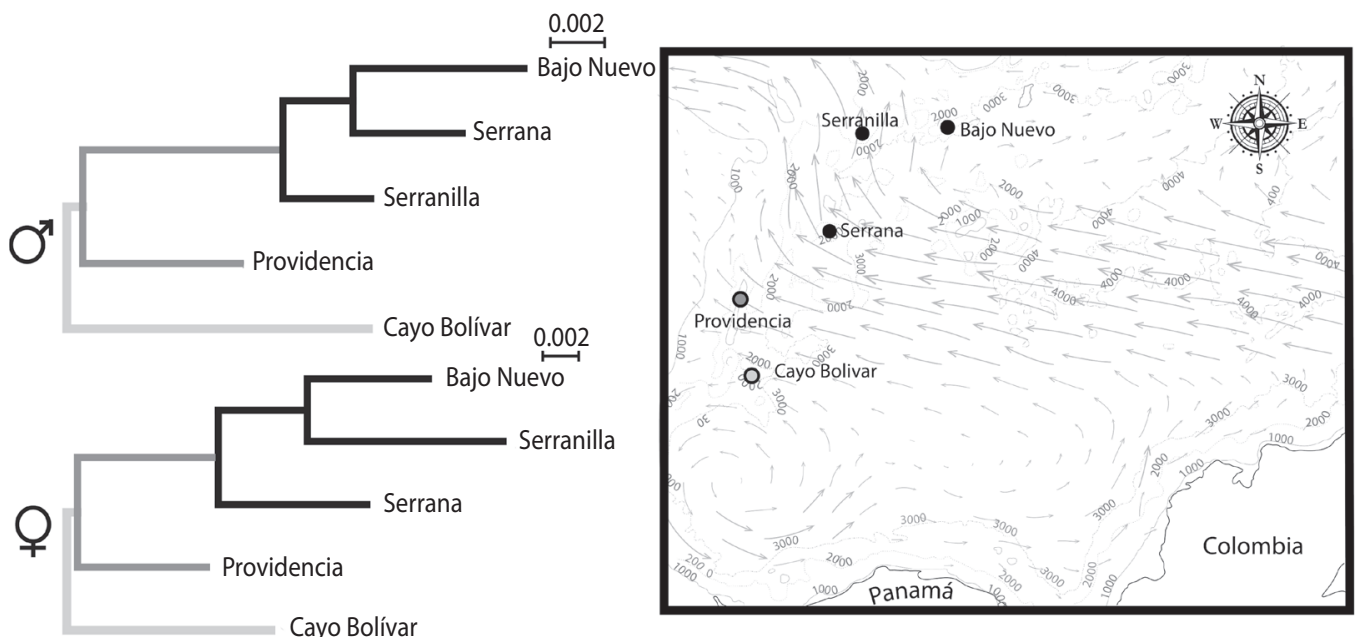

Fig. 6. Árbol Neighbour Joining basado en las distancias Euclidianas entre la conformación de la placa esternal de P. argus del archipiélago San Andrés, Providencia y Santa Catalina.

Fig. 6. Neighbour Joining tree based on Euclidean distances among the sternal plate shape of $P$. argus from the archipelago San Andrés, Providencia and Santa Catalina. 
CUADRO 3

Significancia estadística de las distancias Euclidianas de las conformaciones de las placas esternales de $P$. argus entre sitios de muestreo

TABLE 3

Statistical significance of Euclidian distances of sternal plate shape of $P$. argus among sampling sites

\begin{tabular}{lccccc} 
& Nuevo & Bolívar & Providencia & Serrana & Serranilla \\
Nuevo & & $0.000^{*}$ & $0.001^{*}$ & 0.020 & 0.058 \\
Bolívar & $0.000^{*}$ & & $0.000^{*}$ & $0.000^{*}$ & $0.000^{*}$ \\
Providencia & $0.000^{*}$ & $0.000^{*}$ & & $0.002 *$ & $0.000^{*}$ \\
Serrana & 0.093 & $0.000^{*}$ & $0.000^{*}$ & & $0.000^{*}$ \\
Serranilla & 0.120 & $0.000^{*}$ & $0.002^{*}$ & 0.079 & \\
\hline
\end{tabular}

Diagonal inferior: machos, diagonal superior: hembras. El asterisco denota significancia estadística después de la corrección Bonferroni $(\mathrm{P}<0.0050)$.

Lower diagonal: males, upper diagonal: females. Asterisks denote significant statistical differences after Bonferroni correction $(\mathrm{P}<0.0050)$.

estos factores tienen una influencia estadísticamente significativa sobre el tamaño y la conformación de la placa esternal de la langosta espinosa, lo cual es concordante con la expectativa inicial, y brinda soporte a la hipótesis planteada. El tamaño y la conformación de la placa esternal han sido útiles para discriminar especies crípticas en los complejos Petrolisthes galathinus (Hiller, Kraus, Almon, \& Werding, 2006) y Austropotamobius pallipes (Bertocchi et al., 2008); sin embargo, hasta ahora no había sido utilizada para estudiar la variación fenotípica a nivel micro-evolutivo.

En general, el tamaño de la placa esternal de los machos fue significativamente más grande que el de las hembras, lo cual se ha encontrado en otras estructuras como el caparazón (Gómez, Guzmán, \& Barrios, 2007); pero contrasta con lo observado en otros crustáceos del complejo P. galathinus, Petrolisthes glaselli y Parapetrolisthes tortugensis, en los que las hembras mostraron una placa más ancha que los machos (Hiller et al., 2006). No obstante, el grado de dimorfismo sexual en el tamaño de la placa esternal varió entre localidades del ASPSC, lo cual podría ser producto del bajo número muestral de las hembras en algunos sitios. Sin embargo, no puede descartarse que estas variaciones reflejen plasticidad fenotípica como ocurre en otros taxones (Stillwell, Blanckenhorn, Teder, Davidowitz,
\& Fox, 2010), teniendo en cuenta que diferentes rasgos morfológicos de los crustáceos exhiben plasticidad fenotípica (Lebour, 1928; Guerao, Abelló, \& Dos Santos, 2006; Sato \& Suzuki, 2010; Ogburn, Stuck, Heard, Wang, \& Forward, 2011).

De manera similar, la placa esternal de $P$. argus exhibió dimorfismo sexual en la conformación, aspecto descrito en otras estructuras de crustáceos tales como el caparazón en $C$. maenas (Ledesma et al., 2010), en A. marginata (Trevisan et al., 2012), en el langostino colorado M. rugosa (Claverie \& Smith, 2010) y en los pleópodos de estados juveniles de L. depurator (Guerao \& Abelló, 2011). Este dimorfismo podría resultar de respuestas sexualmente variables a diferentes presiones de selección natural, como ocurre en otras especies como C. maenas, en las que las fuerzas de selección parecen favorecer la conformación de caparazones lateralmente más comprimidos, aumentando la convexidad del caparazón de la hembra (Ledesma et al., 2010).

En este trabajo, se determinó que la placa esternal de las hembras está más ensanchada en la región donde se inserta el cuarto par de patas caminadoras (séptimo esternito torácico); probablemente, para dar espacio suficiente a la espermateca, porque las aperturas gonadales de la hembra se localizan en la coxa del tercer par de pereiópodos (sexto esternito torácico). 
El ensanchamiento podría estar asociado a la capacidad de las hembras de cargar más esperma y la fecundación de los huevos. Estas diferencias sexuales en la conformación de la placa esternal no pueden atribuirse al tamaño, dado que persistieron después de eliminar el efecto alométrico. Lo anterior contrasta con lo observado en otras estructuras en varias especies de crustáceos (Harnoll, 1982; Hines, 1982), $P$. inflatus (García-Rodríguez et al., 2004) y $A$. marginata (Trevisan et al., 2012), donde las limitaciones alométricas parecen modular la fecundidad de las hembras (Hines, 1982; Trevisan et al., 2012).

Adicionalmente, en este trabajo se encontraron diferencias en el tamaño y conformación de la placa esternal de la langosta espinosa entre los diferentes sitios examinados. Estos resultados no se explican por el método de captura, el cual fue el mismo en todos los sitios, ni por diferencias genéticas debido a la ausencia de estructuración entre langostas provenientes de los sitios estudiados (J. D. Rangel-Medrano, comunicación personal, diciembre 15 de 2015). Los resultados tampoco parecen explicarse por la presión de pesca por la ausencia de una relación directa con el tamaño de los individuos (excepto en Providencia). La relación entre disminución del tamaño del cuerpo y la talla de madurez sexual como resultado del efecto de la pesca se ha observado en $P$. argus (Gómez et al., 2007) y en el cangrejo Goniposis cruentata (Hirose et al., 2015).

Las conformaciones de las placas esternales de $P$. argus fueron diferentes entre algunos sitios y mostraron una tendencia de agrupación con el escenario oceanográfico de las zonas norte, centro y sur del ASPSC, lo cual es concordante con lo encontrado en otra especie de la misma área geográfica (E. J. Márquez, comunicación personal, diciembre 15 de 2015). Las diferencias entre regiones también se han registrado en diversos caracteres morfológicos en $H$. americanus en Nueva Inglaterra (Cadrin, 1995) y Canadá (Harding et al., 1993) y en otros crustáceos como C. maenas (Souza et al., 2011) y C. affinis (Lezcano et al., 2012). Sin embargo, otros trabajos solo han determinado diferencias morfológicas pequeñas entre poblaciones de la langosta europea H. gammarus entre nueve localidades de Reino Unido (Debuse et al., 2001).

Dado que el fenotipo es producto del efecto individual y conjunto del genotipo y el ambiente, la evidencia de flujo génico entre los atolones del ASPSC (J. D. Rangel-Medrano, comunicación personal, diciembre 15 de 2015) apoya la idea, que las poblaciones de langosta genéticamente similares exhiben variaciones fenotípicas como posible respuesta a la heterogeneidad ambiental. Además, es razonable pensar que la principal estrategia evolutiva para aumentar la adaptación local de la langosta es la plasticidad fenotípica, teniendo en cuenta que su dispersión amplia durante el desarrollo larvario le permite experimentar diferentes ambientes, mientras que en una etapa juvenil y adulta experimenta un ambiente más homogéneo y relativamente sedentario, por las condiciones batimétricas del ASPSC. El razonamiento anterior está basado en la idea que la plasticidad fenotípica es la estrategia principal para facilitar la adaptación local en organismos que cambian el potencial de dispersión a lo largo de su vida (Van Tienderen, 1991; DeWitt, Sih, \& Wilson, 1998).

La plasticidad fenotípica como estrategia para responder a los ambientes heterogéneos también se ha descrito en $M$. gregaria en respuesta a señales de asentamiento larvario (Chilton, 1909) o a cambios en la temperatura del agua relacionados con la profundidad (Bacardit, 1986). Se ha propuesto que estas variaciones fenotípicas pueden explicar la existencia de dos morfotipos de $M$. gregaria: gregaria y subrugosa (Pérez-Barros et al., 2011). La plasticidad fenotípica también se ha descrito en C. maenas (Brian, Fernandes, Ladle, \& Todd, 2006; Silva et al., 2010) como posible respuesta a la presión de depredadores durante su etapa de post-larva (Moksnes, Pihl, \& Van Montfrans, 1998).

Uno de los factores ambientales que puede estar influenciando la variación fenotípica de la placa esternal de las langostas del archipiélago es la densidad poblacional. Las diferencias de 
conformación entre escenarios oceanográficos mostraron en ambos sexos, un gradiente en el que la región anterior de la placa esternal (puntos de inserción de los pares de patas caminadoras 2 y 3 ) se va estrechando a medida que se avanza en el sentido de las zonas sur-norte. Este gradiente parece estar relacionado negativamente con la densidad poblacional de las zonas: las langostas con placas esternales más anchas están en la región de menor densidad poblacional, en donde se espera menor competencia por espacio y alimento; mientras que las langostas con placas más estrechas están en la región de mayor densidad poblacional en donde se espera que dicha competencia sea mayor. Este efecto de densidad poblacional también parece explicar las diferencias observadas en la conformación del caparazón de $C$. maenas (Souza et al., 2011). Alternativamente, la presión de pesca podría explicar el patrón de variación de la conformación de la placa esternal, teniendo en cuenta su relación inversa con la intensidad en las actividades pesqueras en estas zonas del ASPSC. Sin embargo, se carece de información y procedimientos estandarizados que permitan hacer comparaciones con los resultados de este trabajo, razón por la cual se requerirán estudios adicionales para contrastar estas hipótesis.

Resulta claro que de acuerdo con los argumentos de Cadrin (2005), en el ASAPSC existen por lo menos tres grupos de langostas con dinámicas de crecimiento distintas (stocks fenotípicos) los cuales deben ser modulados y manejados separadamente, independientes de su homogeneidad genética. La anterior recomendación se hace con base en la idea de que las tasas ontogénicas influencian muchos atributos de la población que pueden estar íntimamente relacionadas a la dinámica poblacional, lo cual determina cómo cada stock responde a la explotación (Garrod \& Horwood, 1984).

En conclusión, los resultados de este estudio indican que la placa esternal de $P$. argus puede variar en el nivel intra-específico de una manera dependiente del sexo y la geografía relacionada con el escenario oceanográfico. Tales variaciones parecen explicarse por plasticidad fenotípica en respuesta a la variación ambiental, reflejando dinámicas de crecimiento distintas en las zonas norte, centro y sur del ASAPSC. Esta información, en conjunto con los resultados genéticos desarrollados para la región (J. D. Rangel-Medrano, comunicación personal, diciembre 15 de 2015), indican que las langostas de este sector del Caribe requieren un esquema de manejo regional dada su alta conectividad genética (varios países) y un manejo local diferencial en el archipiélago de ASPSC (zonas norte, centro y sur).

\section{AGRADECIMIENTOS}

Las expediciones científicas a las zonas remotas de la Reserva de Biosfera Seaflower se realizaron gracias al apoyo de la Corporación para el Desarrollo Sostenible del Archipiélago de San Andrés, Providencia y Santa Catalina - CORALINA, Secretaria de Agricultura y Pesca del Departamento Archipiélago y Universidad Nacional de Colombia Sedes Medellín y Caribe.

\section{RESUMEN}

La langosta espinosa Panulirus argus es una importante especie pesquera en el Atlántico centro-occidental. Los cambios en el rango de dispersión a través de su ciclo de vida y sus hábitats heterogéneos, sugieren que $P$. argus exhibe plasticidad fenotípica. Sin embargo, la variación morfométrica de esta especie es desconocida hasta ahora, aún cuando esta información es relevante en estudios evolutivos y en la solución de preguntas de interés pesquero. Por lo tanto, el objetivo de este estudio fue determinar si $P$. argus exhibe variación fenotípica entre sexos, entre cinco orígenes geográficos y tres condiciones oceanográficas del Caribe suroccidental (archipiélago colombiano de San Andrés, Providencia y Santa Catalina). Un total de 193 adultos de $P$. argus se analizaron por Morfometría Geométrica usando seis puntos de referencia que delimitan la mitad de la placa esternal. Las diferencias de tamaño entre sexos y orígenes geográficos, se compararon con las pruebas de Kruskal-Wallis y Mann-Whitney. El efecto alométrico se estimó usando Análisis de Regresión Multivariada, el modelo de pendientes alométricas se contrastó con un Análisis Multivariado de Covarianza y las diferencias de conformación de la placa esternal se exploró usando comparaciones no paramétricas de las distancias Euclidianas y árboles del vecino más cercano. Los resultados mostraron que la variación morfométrica de la placa 
esternal de la langosta espinosa varía de acuerdo al sexo debido a que el dimorfismo sexual fue significativo tanto en el tamaño como en la conformación. En ambos sexos, la conformación de la placa esternal difirió entre escenarios oceanográficos como se evidenció por diferencias significativas entre distancias Euclidianas y su tendencia a agruparse por las secciones norte, centro y sur del archipiélago de San Andrés. Adicionalmente, la variación morfométrica resultante de plasticidad fenotípica a contextos ecológicos variables pueden explicar las diferencias fenotípicas entre poblaciones que son genéticamente similares. Esta información permite definir unidades de manejo, apoya la selección de medidas regulatorias de esta pesquería y complementa los análisis genéticos de la especie en esta región del Caribe.

Palabras clave: morfometría geométrica, morfología geométrica, plasticidad fenotípica, langosta espinosa del Caribe, stock fenotípico, manejo pesquero.

\section{REFERENCIAS}

Abril-Howard, A., Orozco-Toro, C., Bolaños-Cubillos, N., \& Bent-Hooker, H. (2012). Primera aproximación al conocimiento de las comunidades coralinas de los complejos arrecifales de Serranilla, Bajo Alicia y Bajo Nuevo, Colombia, sección norte de la Reserva de Biósfera Seaflower, Caribe Occidental. Revista Marinas y Costeras. Universidad Nacional de Costa Rica, 4, 51-65.

Alencar, C. E. R. D., Lima-Filho, P. A., Molina, W. F., \& Freire, F. A. M. (2014). Sexual shape dimorphism of the mangrove crab Ucides cordatus (Linnaeus, 1763) (Decapoda, Ucididae) accessed through Geometric Morphometric. The Scientific World Journal, 2014, $1-8$

Andrade, C. A. (2001). Las corrientes superficiales en la cuenca de Colombia observadas con boyas de deriva. Revista de la Academia Colombiana de Ciencias Exactas, Físicas y Naturales, 25(96), 321-335.

Andrade-Amaya, C. A. (2012). Oceanografía del archipiélago de San Andrés, Providencia y Santa Catalina. En D. I. Gómez-López, C. Segura-Quintero, P. C. Sierra-Correa y J. Garay-Tinoco (Eds.), Atlas de la Reserva de Biósfera Seaflower. Archipiélago de San Andrés, Providencia y Santa Catalina. Instituto de Investigaciones Marinas y Costeras "José Benito Vives De Andréis” -INVEMAR-y Corporación para el Desarrollo Sostenible del Archipiélago de San Andrés, Providencia y Santa Catalina-CORALINA-. (pp. 53-59). Santa Marta: Serie de Publicaciones Especiales de INVEMAR No. 28

Arango, L., Manrique, M. L., \& Jaimes, J. C. (2001). Reclutamiento de juveniles de la langosta Panulirus argus con refugios artificiales en el Caribe colombiano. Revista de Investigaciones Marinas, 22, 221-228.
Arnqvist, G., \& Märtensson, T. (1998). Measurement error in geometric morphometric: empirical strategies to asses and reduce its impact on measure of shape. Acta Zoologica Academiae Scientiarum Hungaricae, 44, 73-96.

Bacardit, R. (1986). Larvas de Crustácea Decápoda del mar patagónico argentino, incluyendo el talud continental, adyacencias a la Tierra del Fuego e Islas Malvinas. Aspectos morfológicos, ecológicos y filogenéticos. Buenos Aires: Universidad de Buenos Aires.

Bertocchi, S., Brusconi, S., Gherardi, F., Buccianti, A., \& Scalici, M. (2008). Morphometrical characterization of the Austropotamobius pallipes species complex. Journal of Natural History, 42 (31-32), 2063-2077.

Booke, H. E. (1981). The conundrum of the stock concept are nature and nurture definable in fishery science? Canadian Journal of Fisheries and Aquatic Sciences, $38,1479-1480$.

Bookstein, F. L. (1991). Morphometric tools for landmark data. New York: Cambridge University Press.

Brian, J. V., Fernandes, T., Ladle, R. J., \& Todd, P. A. (2006). Patterns of morphological and genetic variability in UK populations of the shore crab, Carcinus maenas Linnaeus, 1758 (Crustacea: Decapoda: Brachyura). Journal of Experimental Marine Biology and Ecology, 329, 47-54.

Cadrin, S. (1995). Discrimination of American lobster (Homarus americanus) stocks off southern New England on the basis of secondary sex character allometry. Canadian Journal of Fisheries and Aquatic Sciences, 52, 2712-2723.

Cadrin, S. (2005). Morphometric landmarks. En S. Cadrin, K. Friedland, \& J. Waldman (Eds.), Stock identification methods (pp. 153-171). Amsterdam: Elsevier.

Castro, E. (2008). Landings control: a potential strategy for the conservation of spiny lobster in the Archipelago of San Andrés, Providencia and Santa Catalina, Colombia. Proceedings of the Gulf and Caribbean Fisheries Institute, 60, 188-192.

Chilton, C. (1909). Crustacea of the subantarctic islands of New Zealand. En C. Chilton (Ed.), The subantarctic islands of New Zealand (pp. 599-613). New Zealand: Philosophical Institute of Canterbury.

Claverie, T., \& Smith, I. (2010). Allometry and sexual dimorphism in the chela shape in the squat lobster Munida rugosa. Aquatic Biology, 8, 179-187.

Cruz, R. (2002). Manual de métodos de muestreo para la evaluación de las poblaciones de langosta espinosa (Documento Técnico de Pesca. No. 399 Roma). Roma: FAO. 
Debuse, V. J., Addison, J. T., \& Reynodls, J. D. (2001). Morphometric variability in UK population of the European lobster. Journal of the Marine Biological Association of the United Kingdom, 81, 369-479.

DeWitt, T. J., Sih, A., \& Wilson, D. S. (1998). Costs and limits of phenotypic plasticity. Trends in Ecology \& Evolution, 13, 77-81.

Dujardin, J. P. (2013). Click package for Windows. Institut de Recherches pour le Développement (IRD. France). Recuperado de http://www.mpl.ird.fr/morphometrics.

FAO. (2001). Report on the FAO/DANIDA/CFRAMP/ WECAFC Regional workshops on the assessment of the Caribbean spiny lobster (Panulirus argus) (FAO Fisheries Report No. 619). Belize City, Belize, 21 April-2 May 1997 y Mérida, Yucatán, México, 1-12 June 1998.

FAO. (2007). Capacidad de pesca y manejo pesquero en América Latina y el Caribe. Instituto de cooperación, Servicios Estratégicos y Documentación (ICSED) - Santiago, Chile. Roma, Italy: Organización de las Naciones Unidas para la Agricultura y la Alimentación.

García-Rodríguez, F., Aguero, J. D. la C., Perez-Enriquez, R., \& MacLeod, N. (2004). Morphometric analysis of population differentiation and sexual dimorphism in the blue spiny lobster Panulirus inflatus (Bouvier 1895) from NW Mexico. En A. M. T. Elewa (Ed.), Morphometrics: Aplications in Biology and Paleontology (pp. 29-43). Verlag: Springer.doi: 10.1007/978-3-662-08865-4_3.

Garay, J., Castillo, F., Andrade, C., Aguilera, J., Niño, L., De la Pava, M., López, W., \& Márquez, G. (1988). Estudio oceanográfico del área insular y oceánica del Caribe colombiano - Archipiélago de San Andrés y Providencia y cayos vecinos. Boletín Científico $\mathrm{CIOH}, 9,3-73$.

Garrod, J. D. \& Horwood, J. W. (1984). Reproductive strategies and the response to exploitation. En G. W. Potts and R. J. Wootton (Eds.), Fish Reproduction (pp. 367-384). New York: Academic Press.

Goldstein, J. S. (2004). Puerulus Identification Project (Project PIP): "Connecting the Dots" Throughout the Caribbean. Proceedings of the Gulf and Caribbean Fisheries Institute, 57, 833-846.

Gómez, G., Guzmán, R., \& Barrios, A. (2007). Aspectos biométricos y dinámica reproductiva de la langosta espinosa, Panulirus argus, en áreas de pesca de la costa norte de la Península de Paria, estado Sucre, Venezuela. Zootecnia Tropical, 25(3), 6.

Good, P. (2000). Permutation Tests: A practical guide to re-sampling methods for testing hypotheses. New York: Springer.
Goodall, C. R. (1991). Procrustes Methods in the Statistical Analysis of Shape. Journal of the Royal Statistical Society B, 53(2), 285-339.

Guerao, G., \& Abelló, P. (2011). Early juvenile development of mediterranean Liocarcinus depurator (Crustacea: Decapoda: Brachyura: Portunidae). Journal of Natural History, 45(35-36), 2171-2189.

Guerao, G., Abelló, P., \& Dos Santos, A. (2006). Morphological variability of the megalopa of Liocarcinus depurator (Brachyura: Portunidae) in Mediterranean and Atlantic populations. Journal of Natural History, 40(32-34), 1851-1866.

Harding, G., Kenchington, E., \& Zheng, Z. (1993). Morphometrics of American lobster (Homarus americanus) larvae in relation to stock determinations in the Maritimes, Canada. Canadian Journal of Fisheries and Aquatic Sciences, 50, 43-52.

Harnoll, R. G. (1982). Growth. En L. B. Abele, \& D. E. Bliss (Eds.), The biology of Crustacea. Embriology, morphology, and genetics (Vol 2, pp. 11-196). New York: Academic Press.

Hateley, J., \& Sleeter, T. (1992). A biochemical genetic investigation of spiny lobster (Panulirus argus) stock replenishment in Bermuda. Bulletin of Marine Science, 52, 993-1006.

Hiller, A., Kraus, H., Almon, M., \& Werding, B. (2006). Species boundaries based on color pattern, morphology and molecules, and evolutionary interrelationships between this complex and other Porcellanidae (Crustacea: Decapoda: Anomura). Molecular Phylogenetics and Evolution, 40, 547-569.

Hines, A. H. (1982). Allometric constraints and variables of reproductive effort in Brachyuran crabs. Marine Biology, 69, 309-320.

Hirose, G. L., Souza, L. S., Silva, S. L. R., Alves, D. F. R., \& Negreiros-Franzoso, M. L. (2015). Population structure of the red mangrove crab Goniopsis cruentata (Decapoda: Grapsidae) under different fishery impacts: Implications for resource management. Revista de Biología Tropical, 63(2), 443-457.

Holthuis, L. B. (1991). FAO species catalogue. Marine lobsters of the world. An annotated and illustrated catalogue of species of interest to fisheries known to date. FAO Fish Synopsis, 13, 133-139.

Idaszkin, Y., Márquez, F., \& Nocera, A. (2013). Habitatspecific shape variation in the carapace of the crab Cyrtograpsus angulatus. Journal of Zoology, 290(2), 117-126.

Klingenberg, C. P., Barluenga, M., \& Meyer, A. (2002). Shape analysis of symmetric structures: quantifying variation among individuals and asymmetry. Evolution, 56(10), 1909-1920. 
Lebour, M. V. (1928). The larval stages of the Plymouth Brachyura. Proceedings of the Zoological Society of London, 2, 473-560.

Ledesma, F. M., Van der Molen, S., \& Barón, P. J. (2010). Sex identification of Carcinus maenas by analysis of carapace geometrical morphometry. Journal of Sea Research, 63(3-4), 213-216.

Lewis, J. B. (1951). The phyllosoma larvae of the spiny lobster Panulirus argus. Bulletin of Marine Science, 1, 89-103.

Lezcano, A. H., González-José, R., Spivak, E. D., \& Dellatorre, F. G. (2012). Geographic differences in the carapace shape of the crab Cyrtograpsus affinis (Decapoda: Varunidae) and its taxonomic implications. Scientia Marina, 76(2), 329-337.

Márquez, E., Landínez-García, R. M., Ospina-Guerrero, S. P., Segura, J. A., Prada, M., Castro, E., Correa, J. L., \& Borda, C. (2013). Genetic analysis of queen conch Strombus gigas from South West Caribbean. Proceedings of the 65th conference of the Gulf and Caribbean Fisheries Institute, 114-121.

Molares, R., Vanegas, T., Bustamante, J., \& Andrade, C. A., (2004). Aspectos oceanográficos de las aguas sobre la depresión Providencia en mayo de 2004. Boletín Cientifico $\mathrm{CIOH}, 22,11-25$.

Moksnes, P. O., Pihl, L. \& Van Montfrans, J. (1998). Predation on postlarvae and juveniles of the shore crab Carcinus maenas: importance of shelter, size and cannibalism. Marine Ecology Progress Series, 166, 211-225.

Ogburn, M. B., Stuck, K. C., Heard, R. W., Wang, S. Y., \& Forward, Jr. R. B. (2011). Seasonal variability in morphology of blue crab, Callinectes sapidus, megalopae and early juvenile stage crabs, and distinguishing characteristics among co-occurring Portunidae. Journal of Crustacean Biology, 31(1), 106-113.

Pérez-Barros, P., Calcagno, J. A., \& Lovrich, G. A. (2011). Absence of a prezygotic behavioural barrier to gene flow between the two sympatric morphs of the squat lobster Munida gregaria (Fabricius, 1793) (Decapoda: Anomura: Galatheidae). Helgoland Marine Research, 65(4), 513-523.

Prada, M., Castro. E., Mitchell, A., \& Bent, K. (2007). Exploring Relationships Between Abundance of Spiny Lobster and Environmental Variability in the San Andres Archipelago: Implications for Fisheries Management. Proceedings of the 60th Gulf and Caribbean Fisheries Institute, 535-540.

Rice, W. R. (1989). Analyzing tables of statistical tests. Evolution, 43, 223-225.
Richardson, P. L. (2005). Caribbean currents and eddies as observed by surface drifters. Deep-Sea Research II, 2005, 429-463.

Rohlf, F. J. 1990. Rotational fit (Procrustes) methods. En F. J. Rohlf, \& F. L. Bookstein (Eds.), Proceedings of the Michigan Morphometrics Workshop (pp. 227236). Ann Arbor: University of Michigan Museum of Zoology.

Rufino, M., Abelló, P., \& Yule, A. B. (2004). Male and female carapace shape differences in Liocarcinus depurator (Decapoda, Brachyura): An application of geometric morphometric analysis to crustaceans. Italian Journal of Zoology, 71(1), 79-83.

Sánchez, J. A., Pizarro, V., Acosta, A., Castillo, P., Herron, P., Martínez, J. C., Montoya, P., \& Orozco, C. A. (2005). Evaluating coral reef benthic communities in remote Caribbean atolls (Quitasueño, Serrana, And Roncador banks) to recommend marine-protected areas for the Seaflower Biosphere Reserve. Atoll Research Bulletin, 531, 66.

Sato, T., \& Suzuki, N. (2010). Female size as a determinant of larval size, weight, and survival period in the coconut crab, Birgus latro. Journal of Crustacean Biology, 30(4), 624-628.

Silva, I. C., Alves, M. J., Paula, J., \& Hawkins, S. J. (2010). Population differentiation of the shore crab Carcinus maenas (Brachyura: Portunidae) on the southwest English coast based on genetic and morphometric analyses. Scientia Marina, 74(3), 435-444.

Silberman, J. D., Sarver, S. K., \& Walsh, P. J. (1994). Mitochondrial DNA variation and population structure in the spiny lobster Panulirus argus. Marine Biology, 120, 601-608.

Souza, A. T., Ilarri, M. I., Campos, J., Marques, J. C., \& Martins, I. (2011). Differences in the neighborhood: Structural variations in the carapace of shore crabs Carcinus maenas (Decapoda: Portunidae). Estuarine, Coastal and Shelf Science, 95(4), 424-430.

Stillwell, R. C., Blanckenhorn, W. U., Teder, T., Davidowitz, G., \& Fox, C. W. (2010). Sex differences in phenotypic plasticity affect variation in sexual size dimorphism in insects: From physiology to evolution. Annual Review of Entomology, 55, 227-245.

Tavares, M. (2002). Lobster. En K. E. Carpenter (Ed.). The living marine resources of the Western Central Atlantic. Introduction, molluscs, crustaceans, hagfishes, sharks, batoid fishes, and chimaeras (Volume 1, pp. 1-600). Rome: FAO Species Identification Guide for Fishery Purposes and American Society of Ichthyologists and Herpetologists Special Publication No. 5. 
Tidu, C., Sardá, R., Pinna, M., Cannas, A., Meloni, M. F., Lecca, E., \& Savarino, R. (2004). Morphometric relationships of the European spiny lobster Palinurus elephas from northwestern Sardinia. Fisheries Research, 69(3), 371-379.

Trevisan, A., Zanetti Marochi, M., Costa, M., Santos, S., \& Masunari, S. (2012). Sexual dimorphism in Aegla marginata (Decapoda: Anomura). Nauplius, 20(1), $75-86$.

Truelove, N., Box, S., Butler, M., Behringer, D., \& Preziosi, R. (2013). Genetic connectivity of Caribbean spiny lobster, Panulirus argus, between advective and retentive oceanographic environments. Proceedings of the 66th Gulf and Caribbean Fisheries Institute, 280.

Van Tienderen, P. H. (1991). Evolution of generalist and specialist in spatially heterogeneous environments. Evolution, 45, 1317-1331.

Zelditch, M., Donald, L., Swiderski, H., Sheets, D., \& Fink, W. L. (2004). Geometric Morphometrics for Biologists: A Primer. Paris: Elsevier Academic Press. 